\title{
Métrologie de la turbulence et visualisation Exposé de synthèse
}

\author{
Turbulence metrology and visualization \\ Synthesis report
}

O. Leuchter

ONERA

Cet exposé introductif présente l'état actuel des techniques d'analyse expérimentale des écoulements turbulents. Les principales méthodes (thermo-anémométrie à fil chaud. vélocimétrie laser. méthodes optiques de mesure des scalaires, visualisations) sont brièvement décrites en insistant sur les développements les plus récents. Les performances et les limitations inhérentes des méthodes sont discutées et illustrées par des exemples concrets.

This introductory report discusses the current state of experimental techniques relating to turbulent flows. The main methods used (hot wire-anemometry, laser velocimetry, optical measurement techniques for scalars, visualizations) are described briefly, the emphasis being placed on the most recent developments. The performances and inherent limitations of the methods are discussed and illustrated by means of concrete examples.

On se propose, dans cet exposé introductif, de présenter l'état actuel des techniques d'analyse expérimentale des écoulements turbulents. Les principales méthodes (thermo-anémométrie à fil chaud, vélocimétrie laser, méthodes optiques de mesure des scalaires, visualisations) sont brièvement décrites en insistant sur les développements les plus récents. Les performances et les limitations inhérentes des méthodes sont discutées et illustrées par des exemples concrets.

Une des tâches fondamentales de la métrologie de la turbulence (et des visualisations) consiste à fournir les éléments indispensables à la compréhension physique des principaux mécanismes gouvernant les écoulements turbulents. Les progrès récents réalisés dans le domaine de la modélisation mathématique des écoulements turbulents exigent de la métrologie de nouveaux efforts pour permet- tre la vérification, directe ou indirecte, des hypothèses ou des concepts sur lesquels s'appuie la modélisation.

Pour répondre à ces nouvelles exigences d'une modélisation élaborée, basée sur l'approche statistique, la métrologie doit être en mesure de fournir non seulement les valeurs moyennes et fluctuantes des 3 composantes de la vitesse, mais aussi celles des autres grandeurs physiques concernées comme la pression, la masse volumique et les scalaires transportables tels que la température ou la concentration des espèces (humidité p. ex.). Les grandeurs statistiques à prendre en considération dans ce contexte sont, en premier lieu, les moments d'ordre 2, c'est-à-dire les composantes du tenseur de Reynolds, les variances des fluctuations des scalaires et les corrélations vitesse-scalaire, auxquelles s'ajoutent certaines corrélations triples. En outre, il est souvent indispensable de considérer des 
fonctions de répartition spectrale ou les fonctions de corrélation temporelle qui renseignent sur les échelles caractéristiques de la turbulence ou sur l'existence de phénomènes périodiques ou déterministes. Il convient également de souligner l'intérêt de mesures en deux ou en plusieurs points en vue de caractériser plus directement les structures spatiales de la turbulence ou de procéder à des mesures par échantillonnage conditionnel.

La prise en considération de l'ensemble de ces facteurs exige de la métrologie de la turbulence des qualités de haut niveau. Celles-ci concernent en premier lieu la précision, la résolution spatiale, la résolution en temps, la "non-intrusivité », la capacité de résoudre simultanément 3 composantes de la vitesse (avec, le cas échéant, une ou plusieurs grandeurs scalaires). A cela s'ajoutent des exigences relatives à la facilité de la mise en œuvre, la simplicité des étalonnages, la possibilité du traitement numérique des informations et enfin, celles relatives aux faibles coûts de mise en œuvre et d'exploitation de la méthode. En pratique, le meilleur choix de la méthode résultera toujours d'un compromis entre les impératifs de la qualité métrologique et les performances des matériels disponibles, compte tenu de la nature du problème posé.

La technique la plus ancienne et la plus répandue pour l'analyse quantitative de la turbulence est celle de la thermo-anémométrie à fil chaud. La réponse de l'anémomètre dépend, en premier lieu, de la vitesse, mais aussi d'autres paramètres, comme la pression, la température,... etc. Cette circonstance donne à la technique du fil chaud la possibilité d'analyser la turbulence de vitesse et, en écoulement non isotherme, celle de la température.

La plupart des mesures par fil chaud sont effectuées en écoulement isotherme incompressible. Dans ces conditions, l'anémométrie (fonctionnant en général dans le mode «à température constante ») n'est sensible qu'à la vitesse, en module et en direction. La sensibilité directionnelle du fil chaud confère à cette technique la possibilité de résoudre simultanément plusieurs composantes de la vitesse, en disposant plusieurs fils d'orientations différentes sur la même sonde.

La configuration la plus simple d'une sonde à fil chaud est celle dans laquelle un seul fil est disposé perpendiculairement à l'axe de la sonde. Celle-ci ne détecte de ce fait que les fluctuations $u^{\prime}$ de la composante de vitesse parallèle à cet axe, pourvu que la sonde soit orientée dans la direction de l'écoulement moyen et que la turbulence soit peu intense. Si ces conditions ne sont pas satisfaites, la mesure de $u^{\prime}$ risque d'être contaminée par les deux composantes $v$ et $w$ normales à l'axe de la sonde. Une seule composante du tenseur de Reynolds est mesurable avec cette sonde, à savoir $u^{\prime 2}$.

Pour une sonde dont le fil est incliné (à $45^{\circ}$ p. ex.) par rapport à son axe, la mesure contient (dans les mêmes conditions restrictives que ci-dessus), des informations simultanées sur $u$ et sur $v, v$ étant la composante normale à l'axe de la sonde et parallèle au plan des broches. Pour obtenir les 3 tensions de Reynolds $\overline{u^{\prime 2}}, \overline{v^{\prime 2}}$ et $\overline{u^{\prime} v^{\prime}}$ par exemple, il sera nécessaire d'effectuer des mesures dans deux positions de roulis opposées de $180^{\circ}$ et de compléter celles-ci par une mesure avec la sonde à fil droit. On n'accédera à toutes les composantes du tenseur de Reynolds qu'en répétant les mesures un certain nombre de fois pour différents angles de roulis (au minimum 6).
Les sondes à 2 fils croisés (inclinés respectivement à $+45^{\circ}$ et à $-45^{\prime \prime}$ ) fournissent explicitement, pour une seule position de roulis, deux des trois composantes du vecteur vitesse, $u$ et $v$, à partir desquelles on détermine aisément les 3 tensions de Reynolds $\overline{u^{\prime 2}}, \overline{v^{\prime 2}}$ et $\overline{u^{\prime} v^{\prime}}$ ainsi que les moments d'ordre supérieur. Cette sonde est, comme les précédentes, soumise à des conditions restrictives quant à son inclinaison et au taux de turbulence de l'écoulement. Il est à noter que 4 positions angulaires en roulis sont nécessaires pour obtenir la totalité des composantes du tenseur de Reynolds.

Les conditions restrictives limitant l'emploi des sondes précédentes à des écoulements avec faibles taux de turbulence sont fortement atténuées pour les sondes à 3 ou à 4 fils. En effet, l'utilisation de ces sondes permet de déterminer les 3 composantes de vitesse simultanément et de ce fait d'élargir notablement le domaine angulaire de validité, d'accroitre la précision de la mesure et d'augmenter la rapidité d'exécution des essais. Le recours à des techniques digitales pour l'acquisition et le traitement des signaux s'avère dans ce cas pratiquement inévitable.

Pour les sondes à 3 fils, les fils sont généralement disposés perpendiculairement les uns par rapport aux autres, afin d'élargir au maximum le domaine angulaire de validité de la mesure. L'avantage des sondes à 4 fils réside dans la disposition géométrique particulière des fils. Cette sonde se présente en effet comme une superposition de 2 sondes à 2 fils croisés dont les axes sont confondus et dont les plans des broches sont perpendiculaires entre eux. Cette circonstance facilite largement l'interprétation et l'exploitation des mesures.

Il convient de souligner, notamment pour les sondes multifils, l'importance d'un étalonnage précis, non seulement en vitesse, mais aussi et surtout en incidence-dérapage (ou incidence-roulis). Là encore, des techniques digitales sont pratiquement indispensables.

L'utilisation de l'anémométrie à fil chaud, même avec les sondes les plus sophistiquées, est limitée à des régions de l'écoulement où l'angle entre le vecteur vitesse instantané et l'axe de la sonde reste inférieur à une valeur limite dépendant de la géométrie de la sonde. Ceci implique que les régions d'écoulement décollé ne sont pas accessibles par la technique du fil chaud. Des remèdes ont été proposés pour pallier cet inconvénient, comme p. ex. la technique du "fil chaud volant " ("flying hot wire ") ou du fil chaud pulsé, mais leur emploi conduit à une expérimentation relativement lourde. Pour cette raison, l'exploration des écoulements présentant des zones décollées ou des bulbes de recirculation est généralement confiée à des techniques différentes (p. ex. vélocimétrie laser, voir plus loin).

La technique de la thermo-anémométrie par fil chaud permet également, comme indiqué plus haut, d'accéder aux fluctuations de température en écoulement non isotherme. Pour isoler celles-ci, on utilise des fils très fins (de l'ordre du micron et au-dessous) à très faible surchauffe, afin de minimiser la contamination par la vitesse. Le recours au mode opératoire « à courant constant » qui seul permet de fonctionner à très faible surchauffe, nécessite le plus souvent (surtout dans les écoulements à grande vitesse) une compensation du signal, destinée à le corriger des effets d'inertie thermique du fil; la constante de temps correspondante doit être déterminée en chaque point de 
mesure. Des corrections prenant en compte les effets de la conduction thermique des fils vers les broches doivent aussi être apportées à la mesure.

La mesure simultanée des fluctuations de vitesse et de température, permettant en particulier de déterminer les variances et les corrélations température-vitesse, peut être effectuée à l'aide de sondes multifils spéciales. Les moments statistiques peuvent aussi s'obtenir à l'aide de la technique du diagramme des fluctuations qui consiste à soumettre successivement un seul fil chaud à plusieurs taux de surchauffe. La première méthode, associée à des techniques digitales, est de loin la plus performante, car elle permet de mesurer simultanément deux composantes de vitesse et la température. Son emploi est cependant réservé aux écoulements à faible vitesse et de dimensions suffisantes pour être compatibles avec le volume de mesure de la sonde.

En écoulement turbulent compressible, spécialement en supersonique, les mesures par fil chaud sont beaucoup plus délicates et de ce fait relativement rares. Les fluctuations de vitesse s'accompagnent ici de fluctuations de température dues à la compressibilité, que l'on isole au moyen de la technique du diagramme des fluctuations mentionnée plus haut. Le mode de fonctionnement à courant constant, généralement retenu pour cette application, implique une compensation de la réponse du fil; le mode à température constante est aussi parfois utilisé.

La vélocimétrie laser, technique relativement nouvelle par rapport à celle du fil chaud, est maintenant entièrement opérationnelle, même dans sa version la plus récente permettant de mesurer simultanément 3 composantes de la vitesse. Elle doit être considérée comme un outil complémentaire par rapport au fil chaud. Contrairement à ce dernier, elle mesure exclusivement la vitesse de l'écoulement et est insensible à la température. Son principe de mesure est basé sur la présence de particules dans l'écoulement, dont elle mesure le vecteur vitesse instantané. Ceci exige que les particules suivent parfaitement l'écoulement dans ses moindres détails. Le dosage optimal de la taille des particules et de leur concentration dépend de la configuration étudiée et constitue un problème difficile qui n'a pas encore trouvé de solution tout à fait satisfaisante.

La vélocimétrie laser est utilisée avantageusement dans des situations difficilement accessibles par fil chaud, comme les écoulements très chauds ou avec combustion, les écoulements transsoniques et supersoniques, les écoulements avec recirculation. Elle doit, dans ce secteur, sa supériorité par rapport au fil chaud au fait qu'elle est non intrusive et qu'elle n'est pas soumise, comme le fil chaud, à des restrictions relatives à la direction de la vitesse à mesurer. En effet, équipée de cellules de Bragg, elle est en mesure de discerner sans ambiguïté le sens de la vitesse.

La vélocimétrie laser reste cependant d'un emploi assez délicat, notamment dans sa version complète tridimensionnelle, et sa mise en cuvre sur les installations d'essai est en général assurée par des équipes de spécialistes. Son coût élevé constitue également un obstacle sérieux à une utilisation plus généralisée.
Il convient de mentionner, parmi les méthodes optiques non intrusives récemment développées, celles qui permettent de mesurer les fluctuations de masse volumique ou des grandeurs dérivées de celles-ci. Ces méthodes complètent utilement celle de la vélocimétrie laser, notamment dans les écoulements difficlement explorables par d'autres techniques. On peut citer dans ce contexte la méthode de la diffusion Raman spontanée qui donne accès directement à la concentration des espèces dans un mélange gazeux et, dans certains cas, à la masse volumique du mélange. L'avantage de cette technique réside dans la possibilité de la combiner avec la vélocimétrie laser dans le but d'effectuer des mesures simultanées de vitesse et de masse volumique.

La technique de la DRASC (Diffusion Raman Anti-Stockes Cohérente) permet de mesurer les valeurs instantanées de la densité et de la température dans des conditions plus difficiles que dans le cas précédent. Elle est de ce fait bien adaptée à l'étude des gaz chauds fluctuants et en particulier à l'étude des flammes turbulentes.

Dans la technique de la densitométrie de Fourier par laser, on utilise la diffusion Rayleigh d'un rayonnement infrarouge produit par laser. Le signal utile représente la valeur instantanée d'une transformée de 'Fourier spatiale de la densité du fluide. Cette technique apporte également des renseignements utiles sur la structure de la turbulence dans les écoulements à masse volumique variable.

On peut classer parmi les méthodes non intrusives la méthode de l'hygromètre Lyman-alpha qui mesure la concentration instantanée de l'humidité dans l'air. Son principe est basé sur l'absorption par la vapeur d'eau d'un rayonnement ultraviolet centré sur la raie Lyman-alpha de l'hydrogène. La méthode est utilisée pour l'étude des fluctuations turbulentes de la concentration de vapeur d'eau au-dessus des surfaces d'eau, soit en soufflerie, soit dans la couche atmosphérique. La mesure peut être effectuée simultanément avec celle d'un fil chaud, ce qui permet de déterminer les flux turbulents d'humidité.

Les techniques de visualisation prennent une part très active dans l'approfondissement de nos connaissances sur les écoulements turbulents et contribuent de manière efficace à la compréhension physique des mécanismes de base. Les visualisations en tunnel hydrodynamique, bien que en général qualitatives, sont à cet égard d'un très grand secours. D'autre part, le développement récent des techniques de visualisation par plan de lumière laser et le développement simultané des techniques de traitement des images offrent à la visualisation de nouvelles possibilités d'une approche plus quantitative.

Adresse de l'auteur

Monsieur O. Leuchter

Adjoint scientifique au Directeur de l'aérodynamique

ONERA

8. nue des Vertugadins

92190 Meudon 


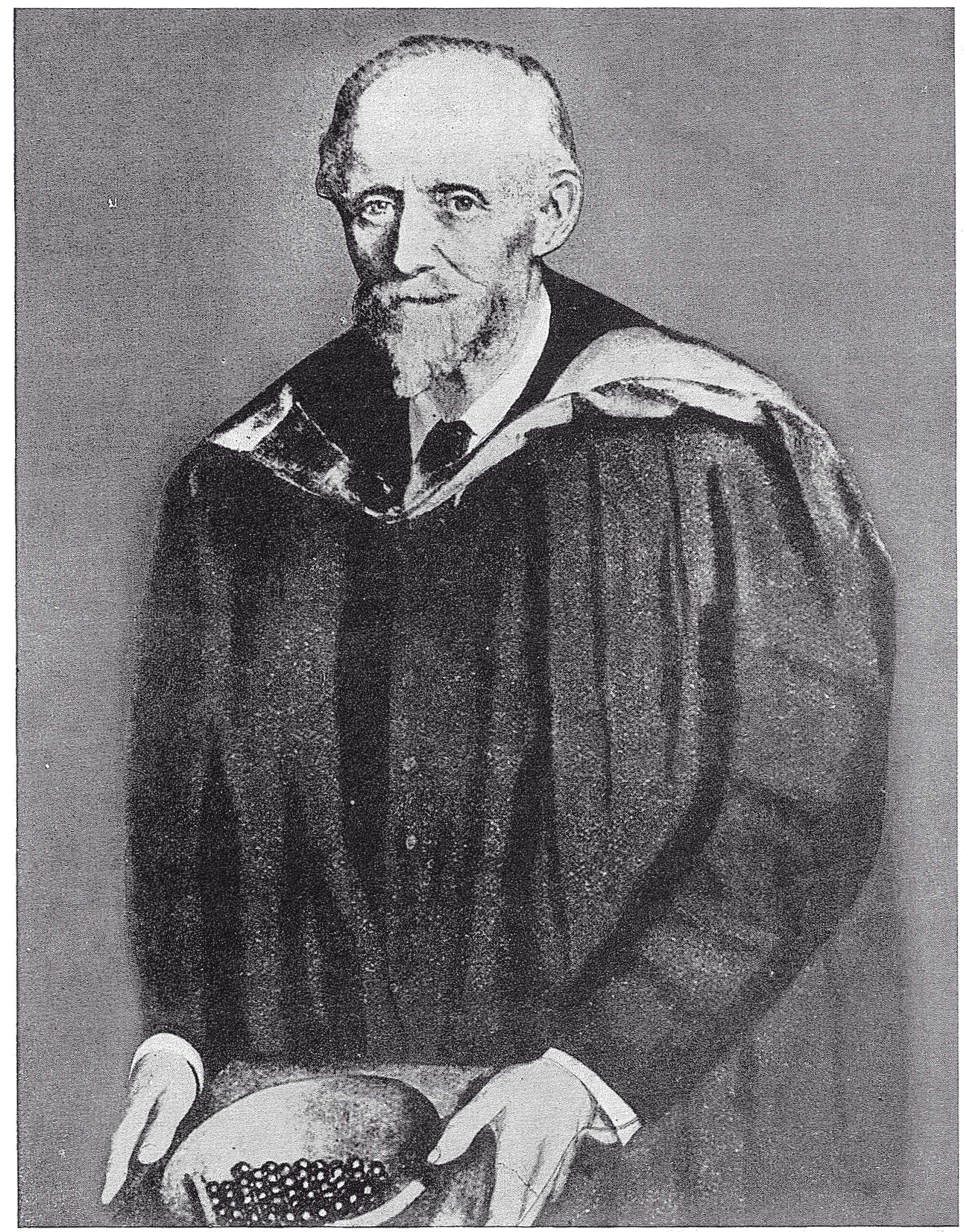

OSBORNE REYNOL.DS (1842-1912) 